\title{
A microquasar model applied to unidentified gamma-ray sources
}

\author{
V. Bosch-Ramon ${ }^{1}$, J. M. Paredes ${ }^{1}$, G. E. Romero ${ }^{2,3, \star}$, and D. F. Torres ${ }^{4}$ \\ 1 Departament d'Astronomia i Meteorologia, Universitat de Barcelona, Av. Diagonal 647, 08028 Barcelona, Spain \\ e-mail: vbosch@am.ub.es; jmparedes@ub.edu \\ 2 Instituto Argentino de Radioastronomía, C.C.5, (1894) Villa Elisa, Buenos Aires, Argentina \\ e-mail: romero@iar.unlp.edu.ar \\ 3 Facultad de Ciencias Astronómicas y Geofísicas, UNLP, Paseo del Bosque, 1900 La Plata, Argentina \\ ${ }^{4}$ Lawrence Livermore National Laboratory, 7000 East Avenue, 413, Livermore, CA 94550, USA \\ e-mail: dtorres@igpp.ucllnl.org
}

Received 21 February 2005 / Accepted 19 September 2005

\section{ABSTRACT}

\begin{abstract}
Among unidentified gamma-ray sources in the galactic plane, there are some that present significant variability and have been proposed to be high-mass microquasars. To deepen the study of the possible association between variable low galactic latitude gamma-ray sources and microquasars, we have applied a leptonic jet model based on the microquasar scenario that reproduces the gamma-ray spectrum of three unidentified gamma-ray sources, 3EG J1735-1500, 3EG J1828+0142 and GRO J1411-64, and is consistent with the observational constraints at lower energies. We conclude that if these sources were generated by microquasars, the particle acceleration processes could not be as efficient as in other objects of this type that present harder gamma-ray spectra. Moreover, the dominant mechanism of high-energy emission should be synchrotron self-Compton (SSC) scattering, and the radio jets may only be observed at low frequencies. For each particular case, further predictions of jet physical conditions and variability generation mechanisms have been made in the context of the model. Although there might be other candidates able to explain the emission coming from these sources, microquasars cannot be excluded as counterparts. Observations performed by the next generation of gamma-ray instruments, like GLAST, are required to test the proposed model.
\end{abstract}

Key words. X-rays: binaries - stars: winds, outflows - gamma-rays: observations - gamma-rays: theory

\section{Introduction}

The instruments EGRET ${ }^{1}$ and COMPTEL $^{2}$, onboard the Compton Gamma Ray Observatory (CGRO), detected about two hundred gamma-ray sources that still remain unidentified. Among these sources, there is a subgroup that appears to be concentrated towards the galactic plane and presents significant variability (Torres et al. 2001; Nolan et al. 2003). The discovery of the microquasar LS 5039, a high-mass X-ray binary (XRB) with relativistic jets, and its association with the high-energy gamma-ray source 3EG J1824-1514 (Paredes et al. 2000), opened the possibility that some other unidentified EGRET sources (Hartman et al. 1999) could also be microquasars. That microquasars can be high-energy gamma-ray emitters has been confirmed by the ground-based Cherenkov telescope HESS, that detected a TeV source whose very small 3- $\sigma$ error box contains LS 5039 (Aharonian et al. 2005). In addition, high-mass microquasars have been proposed to be counterparts of at least a significant fraction of the low galactic latitude unidentified variable EGRET sources (e.g.

\footnotetext{
* Member of CONICET.

1 http//cossc.gsfc.gov/egret/

${ }^{2}$ http//cossc.gsfc.gov/comptel/
}

Kaufman Bernadó et al. 2002; Romero et al. 2004a). Recent statistical and theoretical studies on this group of sources have provided additional support to this association (Bosch-Ramon et al. 2005a). Therefore, it seems at least plausible that microquasars could represent a significant fraction of the variable gamma-ray sources in the galactic plane, generating not only the emission detected by EGRET but also that of variable sources detected by other gamma-ray instruments like COMPTEL. This paper deepens the study of the gammaray source/microquasar connection by applying a detailed microquasar model to three unidentified gamma-ray sources: 3EG J1735-1500 and 3EG J1828+0142, two likely variable unidentified EGRET sources in the galactic plane ${ }^{3}$ (Torres et al. 2001; Nolan et al. 2003), and GRO J1411-64, recently discovered by Zhang et al. (2002) in a re-analysis of the COMPTEL data, which is also both variable and located in the galactic

3 3EG J1735-1500 and 3EG J1828+0142, at galactic latitudes $9^{\circ}$ and $6^{\circ}$ respectively and assuming galactic distances, are at few hundreds of parsecs above the galactic plane. It does not preclude that they are relatively young objects provided that the EGRET microquasar LS 5039 is a runaway object that could during its lifetime reach galactic latitudes up to $10^{\circ}$ or vertical distances of $500 \mathrm{pc}$ from the galactic plane (Ribó et al. 2002). 
plane. Our aim is to check whether a microquasar model "under reasonable assumptions" can be compatible with the observational constraints at different frequencies.

The contents of this paper are arranged as follows: in Sect. 2, the microquasar model is described; in Sect. 3, the application of the model to each source as well as a brief discussion of its results and predictions are presented; the work is summarized in Sect. 4.

\section{The microquasar model}

A semi-analytical model to calculate a microquasar spectrum from radio to gamma-rays has been developed (Bosch-Ramon et al. 2005a). The scenario consists of an X-ray binary system where the compact object, a black hole or a neutron star, surrounded by an accretion disk and a corona, generates collimated outflows or jets (Mirabel \& Rodríguez 1999). The photon fields originating in the companion star and the corona (McClintock \& Remillard 2004) are taken into account. The jet is modeled as an inhomogeneous and magnetized relativistic flow of protons and leptons, and relativistic leptons dominate the radiative processes. Protons will be important dynamically, and this has been taken into account in determining the leptonic luminosity of the jet. This means that the total jet power cannot be less than 10 times the leptonic power, since otherwise the conversion of the jet kinetic luminosity into radiation luminosity probably would be too efficient (see Fender 2001). This fact, related to the macroscopic energy conservation law, imposes that the accretion energy budget should be enough to power the whole jet (as seems to be the case in general, see Bosch-Ramon et al. 2005a). Since it is not clear to what extent they are relevant, we have not accounted for proton radiative properties. We refer to the work of Romero et al. (2003) for the radiative properties of hadronic jets in microquasars.

In this leptonic model, radio emission is generated by an outer jet that expands at a lower velocity than what is expected for the conical case. This type of expansion is introduced to simulate the particle re-acceleration processes allowing extended radio emission (i.e. through bulk motion dissipation of energy caused by external medium interaction, or by instabilities in the flow of internal origin). This radio jet starts where the high energy jet emission is no longer significant, at about 100 times the distance of the jet injection point to the compact object. Other works that have adopted slowly expanding jet models are, for instance, Ghisellini et al. (1985) and Hjellming \& Johnston (1988). In the optical-UV band, emission is in general dominated by the star and, at higher energies, the corona and/or the inner region of the jet. Because of the higher density and pressure conditions than those of radio jets, this inner region is modeled as conical, i.e. undergoing free expansion.

Jet particles interact with the present photon fields (synchrotron, star, accretion disk and corona photons) through the inverse Compton (IC) effect. In our case, the contribution from the disk scattered photons is negligible in front of the corona IC component, since disk photons come from behind the jet and few of the disk scattered photons reach the observer (Dermer et al. 1992). Disk emission itself cannot be particularly

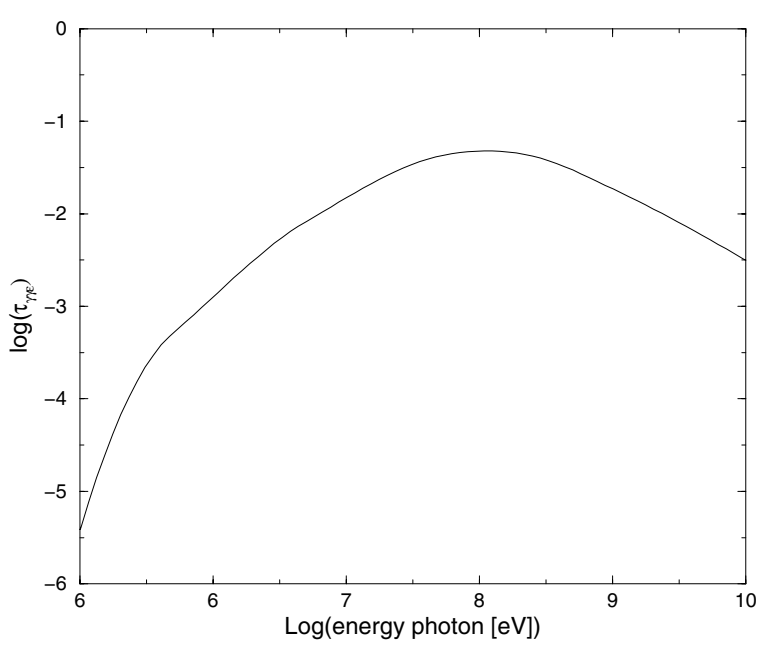

Fig. 1. Opacities at different photon energies in the base of the jet. The dominant corona luminosity has been taken to be $3 \times 10^{34} \mathrm{erg} \mathrm{s}^{-1}$.

significant since it is constrained by the fact that the source remains unidentified at X-rays (see below). Therefore, since this component is superfluous for the modeling, it has not been considered. We have accounted for both Thomson and KleinNishina regimes of IC interaction (Blumenthal \& Gould 1970). The different functions that represent the electron energy distribution, the maximum electron energy and the magnetic field within the jet have been parametrized to simulate their evolution along the jet (e.g., Ghisellini et al. 1985; Punsly et al. 2000).

For this model, the opacities due to pair creation under the jet conditions considered here have been computed and are negligible at this stage. In Fig. 1, the opacities for different energies in the base of the jet, where they are the highest, are shown. The calculation has been performed for a general case, similar however to those treated below. Moreover, the opacity within the stellar photon field at the photon energies involved here is negligible. Nevertheless, for microquasars with spectra extending to $100 \mathrm{GeV}$, or with more luminous corona and/or jet inner regions (e.g. see Romero et al. 2002), opacities would not be negligible.

Our model predicts variability through variations in the leptonic jet power, likely linked to orbital eccentricity and accretion rate changes, as well as to changes in the jet viewing angle due to jet precession. Changes in the jet kinetic luminosity imply an increase of the jet radiating particle density, and precession implies variation in the Doppler boosting that has implications for both the flux and the maximum energy of the observed photons. This issue is discussed qualitatively in Sect. 3.4.

\section{Application of the model to unidentified $\gamma$-ray sources}

In this section, we investigate whether a high-mass microquasar model could reproduce the gamma-ray emission from 3EG J1735-1500, 3EG J1828+0142 and GRO J1411-64, observing other constraints at lower energies. We do not intend either to identify the counterparts or to perform a statistical approach for fitting of our model because only higher resolution gamma-ray observations can solve the identification problem 
and, regarding the latter issue, available data are sparse and a statistical fit would be meaningless. We do not adopt the flux of any particular source in the gamma-ray error boxes as the reference one provided the counterpart is unknown, but typical radio and X-ray fluxes of the sources found inside those fields are taken as upper limits at these energy ranges. If the emission at these frequency bands significantly overcame the typical fluxes found in the gamma-ray error boxes, say, by one order of magnitude, the source would be barely unidentified. Lowerlimits on the fluxes cannot be stated since the counterpart could be relatively quiet in radio and X-rays, being unnoticed by the surveys carried out so far over the regions corresponding to the gamma-ray error boxes. All this implies that the flux can only be constrained roughly.

In the optical band, even though high-mass microquasars have bright stellar companions, clear counterparts have not been found in the gamma-ray error boxes. This could be explained by the strong absorption and/or enshrouding in the optical, UV and even in the X-ray band that is often present towards the galactic plane. For instance, it has been suggested that obscured INTEGRAL ${ }^{4}$ sources could be intrinsically or locally obscured in the UV and X-ray band (e.g. Walter et al. 2003). Furthermore, emission from the massive companion of an X-ray binary scattered and/or reprocessed to the far infrared could even be too weak to be detected by, for instance, the satellite IRAS (e.g. Filliatre \& Chaty 2004). At the adopted distances, the bright companions assumed here would present a relative brightness in the optical band of about $12 \mathrm{mag}$, if not absorbed.

In the absence of specific knowledge, we have fixed the values of the jet parameters entering in the model to fiducial standards for microquasars. For the binary system parameters and jet size, we have adopted those used in Bosch-Ramon et al. (2005a), and a Lorentz factor of 1.2, similar to that shown by the microquasars LS 5039 (3EG J1824-1514, Paredes et al. 2000) and LS I +61 303 (3EG J0241+6103, Kniffen et al. 1997), which present mildly relativistic jets (Paredes et al. 2000; Massi et al. 2004). This should be sufficient, if gammaray microquasars in the low-hard state share similar properties. For the jet viewing angle $(\theta)$, provided again that the jets of LS 5039 and LS I +61 303 are mildly relativistic and it is not required for them to have small $\theta$ to be detected (Paredes et al. 2000; Massi et al. 2004), we have taken a mean value in our specific models of $45^{\circ}$. For the corona spectrum, we have adopted a power-law plus an exponential cut-off with the maximum flux at $100 \mathrm{keV}$. The star has been taken to be a blackbody peaking at UV energies.

The electron energy distribution (assumed to be a powerlaw of index $p$ and starting from energies of about $1 \mathrm{MeV}$ ) and the corona luminosity have been chosen such that they reproduce the gamma-ray spectra and are compatible with the fluxes at lower energies, adopted similar to those inferred from typical sources in the error boxes. As stated above, disk emission itself is limited by the X-ray observational constraints which, together with a lower IC scattering probability (Dermer et al. 1992), makes its IC contribution negligible (for

\footnotetext{
${ }^{4}$ http://integral.esac.esa.int/
}

the assumed viewing angle of $45^{\circ}$, it is almost one order of magnitude smaller than the corona IC contribution). The electron maximum energy together with the magnetic field, given a certain value of $p$, have been taken to reproduce properly the observed spectral slope of gamma-rays. Since the spectral EC components seem to be unable to reproduce the spectrum in gamma-rays, the magnetic field has been taken such that the SSC process is dominant. For instance, if the corona scattered photons were dominant, it would violate the X-ray constraints for any reasonable parameter choice. The leptonic jet power has been taken to obtain the luminosities expected if the sources are at one particular distance (see below). However, the adopted value is similar to those obtained for microquasar jets in previous works using different approaches (e.g. BoschRamon \& Paredes 2004a). Although specific values are provided in Table 1, we give in Sect. 3.4 the set of values for the magnetic field, the jet power and the electron maximum energy that are compatible with data.

The distance from these sources to the Earth has been taken to be $\sim 4 \mathrm{kpc}$. We have assumed that the sources are located close to the inner spiral arms, which have been associated with microquasar birth regions (Bosch-Ramon et al. 2005a). To investigate the variability properties of the studied sources within the context of our model, we have computed the spectral energy distributions (SEDs) associated with the average and the maximum level of the observed gamma-ray fluxes. In the case of the two EGRET sources, the average flux (luminosity) is given by the total exposure EGRET spectrum ${ }^{5}$, and the maximum flux (luminosity) is given by the highest flux among the different EGRET viewing period fluxes (Hartman et al. 1999). To extrapolate fluxes at lower energies, we have assumed that the variations are linked to changes in the accretion rate, linearly related to the jet power, although it is possible to distinguish jet power variations from precession (see Sect. 3.4). For the COMPTEL source, the average value and the maximum one are very similar because actual detections are similar in flux, and the remaining observations only were able to give upper limits for the source (see Zhang et al. 2002).

\subsection{EG J1735-1500}

3EG J1735-1500 was considered in the work of Torres et al. (2001) as a variable EGRET source, and in Nolan et al. (2003) it was also among the group of likely variable EGRET sources (probability $\sim 60 \%$ ). The EGRET spectrum shows a photon index $\Gamma \sim 3.2 \pm 0.5$ and average flux $\sim 3 \times 10^{-11} \mathrm{erg} \mathrm{s}^{-1} \mathrm{~cm}^{-2}$. The error box of 3EG J1735-1500 was explored by Combi et al. (2003), who proposed two potential counterparts: a radio galaxy (J1737-15) and a compact radio source (PMN J1738-1502), a blazar candidate, that presents a flat radio spectrum and flux densities of about $0.3 \mathrm{Jy}$. However, since at the present stage it is still hard to explain both whether a radio galaxy can present the variability of 3EG J1735-1500 and the absence of X-ray counterpart for the compact radio source, we have not considered them as definitive solutions of the identification problem. To model the SED of a microquasar

\footnotetext{
5 http://cossc.gsfc.nasa.gov/compton/data/egret/
} 
Table 1. Common and specific values for the parameters.

\begin{tabular}{cl}
\hline \hline Parameter & Values \\
\hline Stellar bolometric luminosity [erg s & \\
Distance from the apex of the jet to the compact object $[\mathrm{cm}]$ & $5 \times 10^{38}$ \\
Initial jet radius [cm] & $5 \times 10^{6}$ \\
Orbital radius [cm] & $3 \times 10^{12}$ \\
Viewing angle to the axis of the jet $\left[^{\circ}\right]$ & 45 \\
Jet Lorentz factor & 1.2
\end{tabular}

\begin{tabular}{|c|c|c|c|}
\hline & 3EG J1735-1500 & 3EG J1828+0142 & GRO J1411-64 \\
\hline Jet leptonic kinetic luminosity $\left[\mathrm{erg} \mathrm{s}^{-1}\right]$ & $5 \times 10^{34}$ & $10^{35}$ & $3 \times 10^{35}$ \\
\hline Maximum electron Lorentz factor (jet frame) & $3 \times 10^{3}$ & $4 \times 10^{3}$ & $5 \times 10^{2}$ \\
\hline Maximum magnetic field [G] & 10000 & 5000 & 8000 \\
\hline Electron power-law index & 2 & 2 & 1.5 \\
\hline Total corona luminosity [erg s${ }^{-1}$ ] & $3 \times 10^{34}$ & $3 \times 10^{33}$ & $3 \times 10^{33}$ \\
\hline
\end{tabular}

that could be the origin of the EGRET emission, we take into account the known observational data and constraints at different wavelengths. If the distance were $4 \mathrm{kpc}$, the typical luminosities of the radio sources in the EGRET error box would be of about $2 \times 10^{30} \mathrm{erg} \mathrm{s}^{-1}$, the X-ray luminosities would be $\sim 10^{34} \mathrm{erg} \mathrm{s}^{-1}$, and at COMPTEL energies the upper limits would be $\sim 10^{36} \mathrm{erg} \mathrm{s}^{-1}$ (Zhang et al. 2004). The used parameter values are presented in Table 1. The computed SED for both the average and the maximum luminosity levels of the gamma-ray source are shown in Fig. 2. It appears that 3EG J1735-1500, even if detectable at X-rays during its maximum luminosity level, would be faint at radio wavelengths. At optical wavelengths, we have computed the visual extinction of 1.4 mag from the relationship with the hydrogen column density found by Predehl \& Schmitt (1995). It seems from Fig. 2 that additional intrinsic absorption would be necessary to obscure the source in the optical band to prevent an easy identification, since it still has an absolute brightness of 13.4 mag. To reproduce the observed gamma-ray variability through the jet precession, with the adopted mildly relativistic velocity of the jet, the variation in the angle should be large, reaching almost $0^{\circ}$. However, an orbital eccentricity of 0.5 or less could be enough to change the jet power, producing the observed ratio of maximum to average luminosity (see, e.g., Bosch-Ramon et al. 2005b).

\section{2. $3 E G ~ J 1828+0142$}

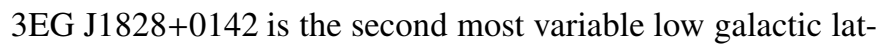
itude non-transient gamma-ray source in the list of variable EGRET sources of Torres et al. (2001), considered also very variable by Nolan et al. (2003). The EGRET photon index is $\Gamma \sim 2.7 \pm 0.4$, with an average flux $\sim 4 \times 10^{-11} \mathrm{erg} \mathrm{s}^{-1} \mathrm{~cm}^{-2}$. Within the error box of this EGRET source, there are several faint non-thermal radio sources with luminosities around $5 \times 10^{30} \mathrm{erg} \mathrm{s}^{-1}$ (Punsly et al. 2000), and X-ray sources (observed by the ROSAT ${ }^{6}$ All Sky Survey) with typical luminosities of about $10^{33} \mathrm{erg} \mathrm{s}^{-1}$. COMPTEL upper limits

\footnotetext{
${ }^{6}$ http://heasarc.gsfc.nasa.gov/docs/rosat/ rosgof.html
}

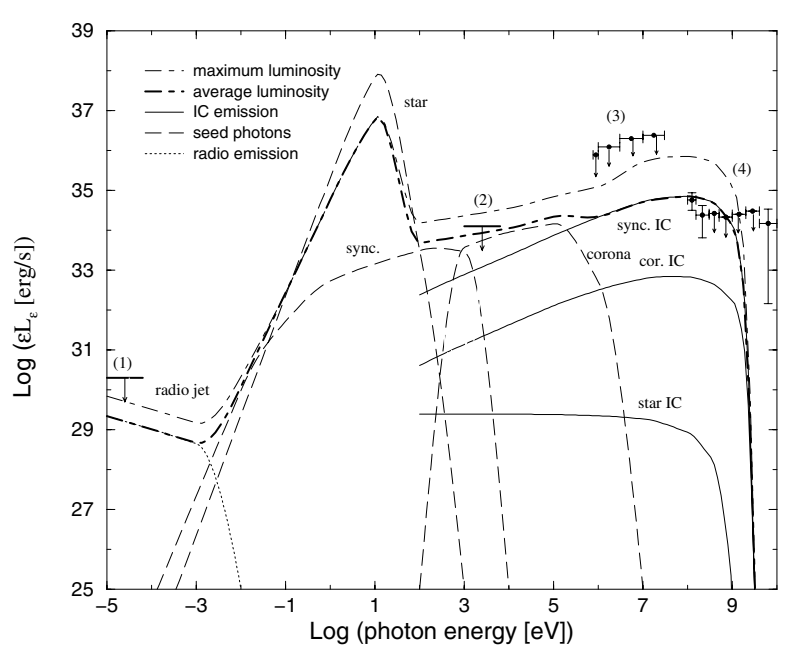

Fig. 2. SED for a broadband microquasar model of the source 3EG J1735-1500. The SED of the average as well as the maximum luminosity level of the source are shown. The adopted values for the different parameters are shown in Table 1. Upper limits at radio (1), X-ray (2) and COMPTEL (3) energies, as well as the average EGRET spectrum (4), are presented. To compute the total emission, the star component has been reduced a certain factor, in accordance to the maximum visual extinction found in the direction of the EGRET source. For the UV, we have followed roughly the relationship between different wavelengths provided by Valencic et al. (2004).

are also known (Zhang et al. 2004), corresponding to luminosities of about $10^{36} \mathrm{erg} \mathrm{s}^{-1}$; the assumed distance still being the same. A supernova remnant (SNR), located at $\sim 1 \mathrm{kpc}$, has been proposed by Punsly et al. (2000) to be associated with the object emitting at gamma-rays. This SNR, yet not part of the Green's Catalog, was not a member of the sample in the systematic study of molecular material by Torres et al. (2003), although the source variability argues against a physical association with the SNR shock. Association with the SNR would imply a lower energy requirement to explain the observed EGRET flux, although with such a distance the source would not be associated with the Carina arm, as most of the EGRET sources in the galactic plane seem to be (Bhattacharya et al. 2003). 


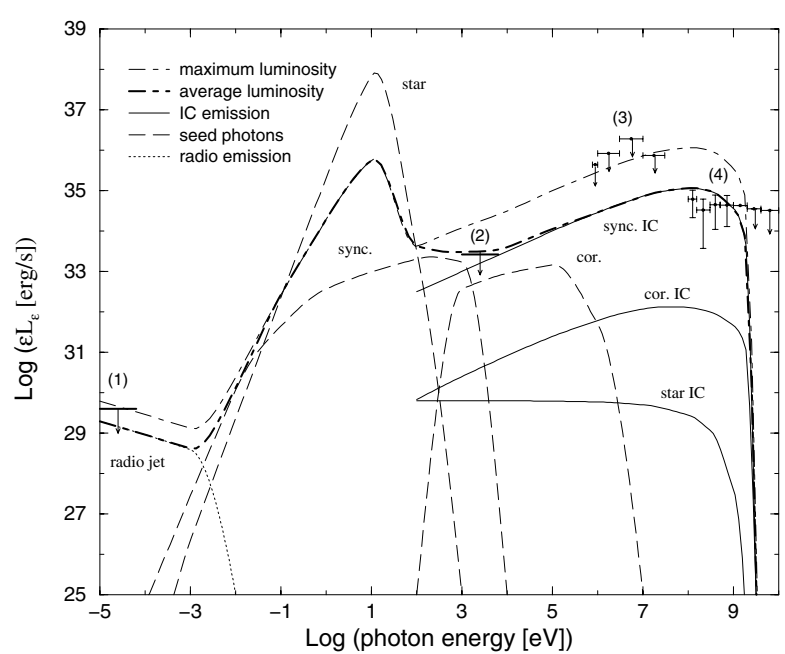

Fig. 3. Same as in Fig. 2 but for 3EG J1828+0142.

Also, there is a strong flat spectrum radio source within the error box of this source which has been proposed to be a blazar (Halpern et al. 2003; Sowards-Emmerd et al. 2003 assigned to the blazar J1826+0149 an association probability of 60-80\%). Further observational data is needed for a firm association of the source with any particular counterpart. The values used for the different parameters are presented in Table 1 and the computed SED for both the average and the maximum luminosity level of the gamma-ray source are shown in Fig. 3. It appears that the X-ray emission of 3EG J1828+0142 could be at the detected source fluxes, and it might be one of the radio sources in the EGRET error box during its most active state. For an absorption of 2.6 mag in the optical band, the optical counterpart would be of a magnitude of about 15 , which makes this source largely irrelevant from the optical point of view among other sources in $1^{\circ}$-field. This will be more so in the ultraviolet, preventing a clear identification. Regarding the variability, the same remarks made for the previous source are applicable to this case, although the ratio of the maximum to average luminosity is slightly smaller and lower eccentricity and/or precession could explain this finding.

\subsection{GRO J1411-64}

The detection by COMPTEL of a variable unidentified gammaray source in the galactic plane, GRO J1411-64, was reported by Zhang et al. (2002). This source has a photon index $\Gamma \sim$ $2.5 \pm 0.2$ and a flux of about $5 \times 10^{-10} \mathrm{erg} \mathrm{s}^{-1} \mathrm{~cm}^{-2}$ at $10 \mathrm{MeV}$. The error box of COMPTEL is large, with a radius of about 2 degrees. Several models have been proposed by Zhang et al. (2002) to explain the gamma-ray emission from this source as a Be/X-ray binary (Romero et al. 2001), a weak galactic microblazar (Kaufman Bernadó et al. 2002) or an isolated black hole (Punsly et al. 2000). Inside the COMPTEL error box, there are only two identified X-ray sources: 2 S 1417-624, a transient Be/XRB pulsar (see Romero et al. 2004b), and GS 1354-645, a transient black-hole low-mass XRB. The remaining detected $\mathrm{X}$-ray sources have no counterparts at other wavelengths. These two XRBs lie just inside the $4 \sigma$ region, and a physical

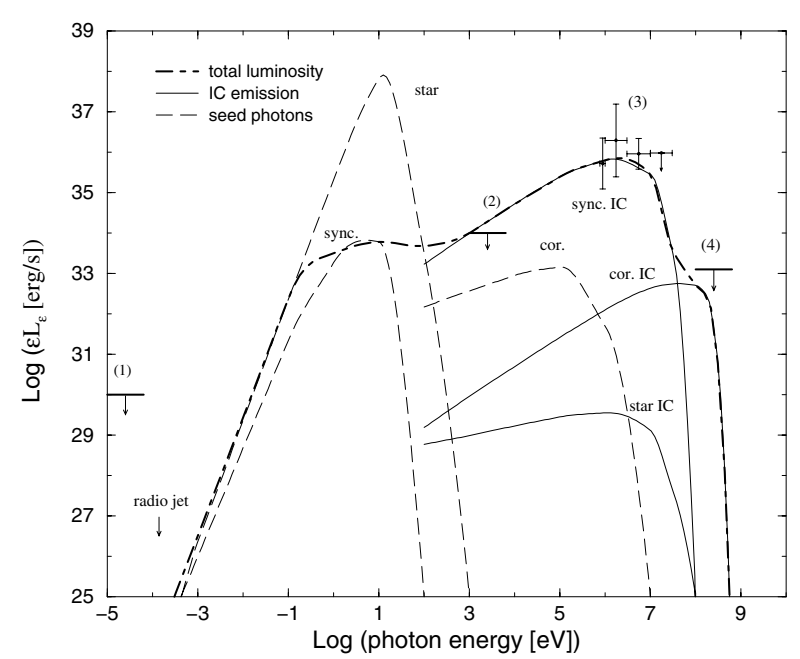

Fig. 4. SED for a broadband microquasar model of GRO J1411-64. The total COMPTEL spectrum (3), the same for the average and the maximum level of emission, as well as the upper limits at radio (1), $\mathrm{X}$-ray (2) and EGRET energies (4). The adopted values for the different parameters are shown in Table 1. The total emission has been reduced in the optical and ultraviolet bands according to the visual extinction in the COMPTEL source direction.

association does not seem likely if the center of gravity of the source location is correct. The Circinus Galaxy is inside the error box though, if there are not ultraluminous gammaray objects in this galaxy, it is unlikely to be the counterpart of GRO J1411-64. In the radio band, the typical flux of the sources found by the PMN survey (Parkes-MIT-NRAO ${ }^{7}$ ) is taken as the upper limit at these wavelengths: a few $10 \mathrm{mJy}$, or about $10^{30} \mathrm{erg} \mathrm{s}^{-1}$. For the constraints on the X-ray flux we will take a luminosity similar to most of the sources detected by ROSAT, i.e. about $10^{34} \mathrm{erg} \mathrm{s}^{-1}$. The distance was assumed to be $4 \mathrm{kpc}$. At high-energy gamma-rays, we will consider the sensitivity limit of EGRET in the region of GRO J1411-64 as the upper limit. For this case, the average flux and the maximum flux observed by COMPTEL are very similar (Zhang et al. 2002). The values used to compute the SED for the different parameters are presented in Table 1 and the SED is shown in Fig. 4. As can be seen, the counterpart might be one of the $\mathrm{X}$-ray sources detected in the COMPTEL error box but its radio emission is too faint for detection. The visual extinction within the COMPTEL error box can reach 7 mag. This could imply that intrinsic absorption is not required to preclude the detection of the optical and ultraviolet counterpart.

\subsection{Implications of the microquasar model}

\subsubsection{Source properties}

Our general conclusions are that, to reproduce the observed soft spectra at gamma-rays, a leptonic radiative process and a low maximum energy for the particles seem to be required. Generally, if the mechanism of emission were hadronic, the spectra would be harder. Moreover, comparing with two microquasar candidates likely to be gamma-ray sources,

\footnotetext{
${ }^{7}$ http://www.parkes.atnf.csiro.au/research/surveys
} 
LS 5039 and LS I +61 303, the electron maximum energies for these two cases (Bosch-Ramon \& Paredes 2004a,b; Aharonian et al. 2005) seem to be significantly higher than for the sources treated here, likely pointing to a more efficient acceleration mechanism. In addition, if the sources were microquasars, the dominant emitting process at high energies likely would be SSC. The dominance of SSC scattering implies that the magnetic field is strong enough to obtain gamma-ray fluxes in agreement with observations and preventing to increase the leptonic jet power to untenable values. This would be the case if the magnetic field were too low and/or the corona scattered photons dominant. Within the context of the model, the values for the magnetic field, the jet power and the maximum electron energy can be restricted to $10000 \mathrm{G}, 10^{35} \mathrm{erg} \mathrm{s}^{-1}$ and $1 \mathrm{GeV}$ respectively. Concretely, the COMPTEL source would present slightly higher jet power and lower maximum electron energy than the two EGRET sources. Otherwise the observed spectrum at gamma-rays could not be reproduced taking into account the observational constraints and the previous theoretical considerations. It is worth noting that these values are coarse estimates of the source properties under a microquasar assumption, not being possible to achieve a better precision because of the lack of knowledge of the counterpart fluxes at low energies. Below $100 \mathrm{keV}$, the spectra must be hard enough to agree with observations. This means that, while for 3EG J1735-1500 and 3EG J1828+0142 an electron powerlaw index of 2 is hard enough, an index of 1.5 is required for GRO J1411-64 to keep the X-ray fluxes to those presented by the sources in the gamma-ray error box. This could be related to a more relativistic shock acceleration in the particle injection of GRO J1411-64, and the lower maximum energy could be associated with stronger losses. We also note that the magnetic field values are 100 times smaller than those of equipartition with relativistic electrons ${ }^{8}$, which is about $10^{6} \mathrm{G}$ for a leptonic jet power of $10^{35} \mathrm{erg} \mathrm{s}^{-1}$. Finally, as noted above, due to the stringent constraints in X-rays, the corona should be faint, which is in agreement with the moderate X-ray emission as well as the lack of clear disk and corona features in the X-ray data of the two likely EGRET microquasars LS 5039 (Bosch-Ramon et al. 2005b) and LS I +61 303 (Leahy et al. 1997).

The radio jets associated with 3EG J1735-1500 and 3EG J1828+0142 could only be detected if the electron energy losses are very low and/or there is re-acceleration, perhaps due to shocks with the ISM at large scales or to internal shocks caused by different flow velocities (Marscher \& Gear 1985). In such a case, in the context of our model, there would be emission at low frequencies (below $1 \mathrm{GHz}$ ) up to large distances (about $1 \mathrm{pc}$ ). To detect it would require an instrument with low angular resolution (about 1 arcmin) and high sensitivity (about $0.1 \mathrm{mJy}$ ). For GRO J1411-64, it seems that radio emission would not be detectable due to the low maximum electron energies and the strong losses in the inner jet. Therefore, these microquasars, in contrast to what is usually expected, would

\footnotetext{
${ }^{8}$ Usually, it is considered to be around equipartition in the inner disk regions. However, this magnetic field is not known at the base of the jet (at $\sim 10^{8} \mathrm{~cm}$ from the compact object). Here it has been treated as a free parameter.
}

not present clear radio jets. Instead, they would present at most diffuse and faint radio lobes.

\subsubsection{Variability}

The two mechanisms of variability that we have studied are leptonic jet power changes, associated with accretion rate changes (e.g. for LS 5039, see Bosch-Ramon et al. 2005b), and precession (e.g. for LS I +61 303, see Massi et al. 2004; for a general case, see Kaufman Bernadó et al. 2002). We note that the plotted maximum luminosity SEDs for 3EG J1735-1500 and 3EG J1828+0142 below gamma-rays correspond to those produced by the variation in the leptonic jet power. However, precession cannot be discounted. 3EG J1735-1500 and 3EG J1828+0142 present average luminosities at gamma-rays that are close to those of their minima (Hartman et al. 1999), which could mean that the peaks are short duration events (e.g. periastron passage of an eccentric orbit or a minimum $\theta$ during the precession of the jet) on the timescales of the EGRET viewing periods (of about two weeks). Instead, GRO J1411-64 shows a long duration burst (Zhang et al. 2002), that could be more associated with a super accretion rate phase than to a persistent jet affected by regular changes of its characteristics. The fact that this source appears to be the brightest, assuming the same distance as for the rest, would give weight to this option.

\subsubsection{Predictions}

In the radio band, a low resolution and high sensitivity instrument would be required to detect 3EG J1735-1500 and 3EG J1828+0142, expecting a very soft spectrum, whereas GRO J1411-64 would not be detectable. If this source is strongly absorbed in the optical and the UV band, it could be still detectable in the infrared band, with higher emission at longer than at shorter wavelengths. However, to test such statement, the location accuracy of the sources should be improved, due to the large number of infrared sources within the gamma-ray error boxes. At X-rays, 3EG J1828+0142 could be detected with reasonable exposure times (e.g. with $\mathrm{XMM}^{9}$ ), whereas 3EG J1735-1500 and GRO J1411-64 would be easily detected due to their higher emission levels at this energy band. For the three sources, the X-ray spectra would present photon indices of 1.5 or less. We note that XMM and INTEGRAL observations of GRO J1411-64 are underway, and we will report on them elsewhere. Observations with the next generation gamma-ray instruments are fundamental to properly associate the gamma-ray sources with their counterparts at lower energies. In the COMPTEL energy range, 3EG J1735-1500 and 3EG J1828+0142 might be detected, at the adopted distance of $4 \mathrm{kpc}$, with an instrument 1-2 orders of magnitude more sensitive than COMPTEL. In the EGRET energy range, GRO J1411-64 might be detected by GLAST $^{10}$ with reasonable exposure times, if observed during an activity period similar to that presented during COMPTEL observations.

\footnotetext{
9 http://xmm.vilspa.esa.es/

10 http://glast.gsfc.nasa.gov/ssc/
} 
Due to the very steep cut-offs at energies beyond $1 \mathrm{GeV}$ for 3EG J1735-1500 and 3EG J1828+0142, and beyond $100 \mathrm{MeV}$ for GRO J1411-64, these sources would not be detected by the new Cherenkov telescopes, although it does not prevent the detection of other microquasars with higher maximum electron energies and/or more beaming. Finally, there are different observational features depending on the dominant variability mechanism. A precessing jet would likely show a periodic variation of both the photon index and the maximum detectable energy at gamma-rays. Moreover, the corona would not suffer variations and gamma-rays (SSC) and radio (synchrotron) emission would vary in the same manner (Dermer et al. 1995). If accretion is the origin of variability, SSC emission mechanism will imply a different response to accretion changes than that presented at X-rays when dominated by the corona. In both cases, however, if our microquasar hypothesis is true, 3EG J1735-1500 and 3EG J1828+0142 variability should be periodic.

\section{Summary}

A microquasar model is applied to model the emission at different wavelengths coming from the direction of 3EG J1735-1500, 3EG J1828+0142 and GRO J1411-64. In the context of this model, the gamma-ray emitting jets would radiate mainly via SSC, and would present a lower electron maximum energy than the microquasars LS 5039 and LS I +61 303. Due to the low electron maximum energy, the radio emission is low, and only detectable for the two EGRET sources at low frequencies if the electrons are effectively re-accelerated in the radio jet, which is expected to be quite extended due to the low radiative efficiency for the electron energy and magnetic field values in there. For the COMPTEL source, detectable radio emission is not expected. We have estimated under what conditions the variability could be produced in the context of both precession and/or eccentric orbit effects, although a scenario where both effects are present seems likely.

Acknowledgements. We thank Dr. Isabelle Grenier, referee of this paper, for useful comments and suggestions that have significantly improved this work. We are grateful to Marc Ribó for his useful comments. V.B-R. and J.M.P. acknowledge partial support by DGI of the Ministerio de Educación y Ciencia (Spain) under grant AYA-2004-07171-C02-01, as well as additional support from the European Regional Development Fund (ERDF/FEDER). During this work, V.B-R has been supported by the DGI of the Ministerio de (Spain) under the fellowship BES-2002-2699. G. E. R is supported by Fundación Antorchas and the Argentine Agencies CONICET and ANPCyT (PICT03-13291). The work of DFT was performed under the auspices of the US DOE (NNSA), by UC's LLNL under contract No. W-7405-Eng-48.

\section{References}

Aharonian, F., Akhperjanian, A. G., Aye, K. M., et al. 2005, Science, 309,746
Bhattacharya, D., Akyüz, A., Miyagi, T., Samimi, J., \& Zych, A. 2003, A\&A, 404, 163

Blumenthal, G. R., \& Gould, R. J. 1970, Rev. Mod. Phys., 42, 237

Bosch-Ramon, V., \& Paredes, J. M. 2004a, A\&A, 425, 1069

Bosch-Ramon, V., \& Paredes, J. M. 2004b, A\&A, 417, 1075

Bosch-Ramon, V., Romero, G. E., \& Paredes, J. M. 2005a, A\&A, 429, 267

Bosch-Ramon, V., Paredes, J. M., Ribó, M., et al. 2005b, ApJ, 628, 388

Combi, J. A., Romero, G. E., Paredes, J. M., Torres, D. F., \& Ribó, M. 2003, ApJ, 588, 731

Dermer, C. D., Schlickeiser, R., \& Mastichiadis, A. 1992, A\&A, 256, L27

Dermer, C. D. 1995, ApJ, 446, 63

Fender, R. P. 2001, MNRAS, 322, 31

Filliatre, P., \& Chaty, S. 2004, ApJ, 616, 469

Ghisellini, G., Maraschi, L., \& Treves, A. 1985, A\&A, 146, 204

Halpern, J. P., Eracleous, M., \& Mattox, J. R. 2003, AJ, 125, 572

Hartman, R. C., Bertsch, D. L., Bloom, S. D., et al. 1999, ApJS, 123, 79

Hjellming, R. M., \& Johnston, K. J. 1988, ApJ, 328, 600

Kaufman Bernadó, M. M., Romero, G. E., \& Mirabel, I. F. 2002, A\&A, 385, L10

Kniffen, D. A., Alberts, W. C. K., Bertsch, D. L., et al. 1997, ApJ, 486, 126

Leahy, D. A., Harrison, F. A., \& Yoshida, A. 1997, ApJ, 475, 823

Marscher, A. P., \& Gear, W. K. 1985, A\&A, ApJ, 298, 114

Massi, M., Ribó, M., Paredes, J. M., et al. 2004, A\&A, 414, L1

McClintock, J. E., \& Remillard, R. A. 2004, to appear as Chapter 4 in Compact Stellar X-ray Sources, ed. W. H. G. Lewin, \& M. van der Klis (Cambridge University Press) [arXiv:astro-ph/0306213]

Mirabel, I. F., \& Rodríguez, L. F. 1999, ARA\&A, 37, 409

Nolan, P. L., Tompkins, W. F., Grenier, I. A., \& Michelson, P. F. 2003, ApJ, 597, 615

Paredes, J. M., Martí, J., Ribó, M., \& Massi, M. 2000, Science, 288, 2340

Predehl, P., \& Schmitt, J. H. M. M. 1995, A\&A, 293, 889

Punsly, B., Romero, G. E., Torres, D. F., \& Combi, J. A. 2000, A\&A, 364,552

Ribó, M., Paredes, J. M., Romero, G. E., et al. 2002, A\&A, 384, 954

Romero, G. E., Kaufman Bernadó, M. M., Combi, J. A., \& Torres, D. F. 2001, A\&A, 376, 599

Romero, G. E., Kaufman Bernadó, M. M., \& Mirabel, I. F. 2002, A\&A, 393, L61

Romero, G. E., Torres, D. F., Kaufman Bernadó, M. M., \& Mirabel, I. F. 2003, A\&A, 410, L1

Romero, G. E., Grenier, I. A., Kaufman Bernadó, M. M., Mirabel, I. F., \& Torres, D. F. 2004a, ESA-SP, 552, 703

Romero, G. E., Orellana, M., \& Torres, D. F. 2004b, ESA-SP, 552, 707

Sowards-Emmerd, D., Romani, R. W., \& Michelson, P. F. 2003, ApJ, 590, 109

Torres, D. F., Romero, G. E., Combi, J. A., et al. 2001, A\&A, 370, 468

Torres, D. F., Romero, G. E., Dame, T. M., et al. 2003, Phys. Rept., 382, 303

Valencic, L. A., Clayton, G. C., \& Gordon, K. D. 2004, ApJ, 616, 912

Walter, R., Rodriguez, J., Foschini, L., et al. 2003, A\&A, 411, 427

Zhang, S., Collmar, W., \& Schönfelder, V. 2002, A\&A, 396, 923

Zhang, S., Collmar, W., Hermsen, W., \& Schönfelder, V. 2004, A\&A, 421,983 